\title{
Optical Properties of Nanostructure CuO Prepared by Different Stabilizing Agents
}

\author{
M. A. El-Sherbiny*, K. Abdullah, and S.A. El-Khodary ${ }^{+}$ \\ Department of physics, Faculty of science, Al-Azhar University, Nasr city \\ 11884 Cairo, Egypt. \\ ${ }^{+}$Housing and Building National Research Center (HBRC), Dokki \\ 12311, Giza, Egypt.
}

The effect of using different stabilizing agents for the synthesis of $\mathrm{CuO}$ nanocrystals on the morphological structure was studied. Spec grad $\mathrm{CuCl}_{2}$ and three different stabilizing agents $\mathrm{LiOH}, \mathrm{NaOH}$ and $\mathrm{kOH}$ were used as starting materials. X-ray powder diffraction (XRD), transmission electron microscopy (TEM), selected area electron diffraction (SAED), and UV-Visible absorption spectroscopy were used for characterizing the obtained powder samples. Surface morphologies of the obtained nanorods were found to depend on the used stabilizer. The breadth and length of the obtained nanorods were also estimated. The optical band gap of the prepared samples were found to be 2.09, 2.28, and $2.64 \mathrm{eV}$ for $\mathrm{LiOH}, \mathrm{NaOH}$ and $\mathrm{kOH}$ respectively.

\section{Introduction}

It was established that, nanomaterial properties (mechanical, physical, chemical, electronic, optical and magnetic) differ from that of bulk counterparts. This is due to the high surface area to volume ratio as well as quantum confinement effects [1]. Nanoparticles exist in several different morphologies such as spheres, cylinders, rods, platelets, tubes, etc. Generally, they are designed with surface modifications tailored to meet the needs of given applications they are going to be used for [2]. Oxides of transition metals are important class of semiconductors, because of their wide range of applications: magnetic storage media, solar energy transformation, electronics and catalysis [3]. Cupric oxide $(\mathrm{CuO})$ is a p-type semiconducting oxide with monoclinic crystal structure and an indirect band gap of $\sim 1.2 \mathrm{eV}$ with interesting electrochemical, photovoltaic, and catalytic properties. Various applications have been reported for nanostructured $\mathrm{CuO}$ such as heterogeneous catalysis, solar cells, gas sensors and magnetic storage media where it offers highly reactive surfaces, and improved optical, electrical, and catalytic properties compared to that of bulk crystals. Recently, 
the controlled shape synthesis of nanostructured $\mathrm{CuO}$ has attracted considerable attention where variety of $\mathrm{CuO}$ nanostructures (e.g. nanoneedles, nanoribbons, nanowires, nanorods, and nanosheet configurations) have been fabricated by pulsed laser ablation, sol-gel, hydrothermal processing and thermal oxidation [4]. It was reported that there are different parameters such as temperature, time, $\mathrm{pH}$ value, pressure, concentration of chemical species and capping agent influence the morphologies of nanocrystal $[5,6]$. In the present article different stabilizing agent were used in order to study the effect of solute additions on the properties of the produced nanoparticles. The $\mathrm{CuO}$ nanostructure samples were obtained using different stabilizing agents of $\mathrm{LiOH}, \mathrm{NaOH}$ and $\mathrm{kOH}$.

\section{Experimental}

\subsection{CuO Nanoparticles Preparation}

Three powder samples of nanocrystalline $\mathrm{CuO}$ were prepared by dissolving $0.07 \mathrm{M}$ of cupper chloride (S D Fine Chem. Limited, India) in $50 \mathrm{ml}$ of distilled water under vigorous stirring for 20 minutes. Alkali solution of $(\mathrm{X}) \mathrm{OH}$ were added to the $\mathrm{Cu}$ solution drop by drop with constant stirring at $57^{\circ} \mathrm{C}$ until the $\mathrm{pH}$ value reaches 9 according to equation (1).

$$
\mathrm{CuCl}_{2}+2 \mathrm{XOH} \rightarrow \mathrm{Cu}(\mathrm{OH})_{2} \downarrow+2 \mathrm{XCl}
$$

where $(\mathrm{X})$ is $\mathrm{Li}, \mathrm{Na}$ and $\mathrm{k}$.

With the aid of hydrothermal process, part of the $\mathrm{Cu}(\mathrm{OH})_{2}$ colloids dissociate into $\mathrm{Cu}^{2+}$ and $(\mathrm{OH})^{-}$ions, and when the concentration of $\mathrm{Cu}^{2+}$ and $(\mathrm{OH})^{-}$reaches the supersaturation $\mathrm{CuO}$ nuclei will form according to the following reactions:

$$
\begin{aligned}
& \mathrm{Cu}(\mathrm{OH})_{2} \leftrightarrow \mathrm{Cu}^{2+}+2(\mathrm{OH})^{-} \\
& \mathrm{Cu}^{2+}+2(\mathrm{OH})^{-} \leftrightarrow \mathrm{CuO}+\mathrm{H}_{2} \mathrm{O}
\end{aligned}
$$

The obtained products were separated by centrifugation and washed thoroughly by distilled water, absolute ethanol and acetone in sequence to remove the possibly remaining ions. The ingots then dried in air at room temperature.

\subsection{Analytical Techniques.}

The structure of the prepared samples were investigated (XRD) using (Philips PW3050/60, MPSS) diffractometer, with nickel- filtered $\mathrm{Cu}-\mathrm{K} \alpha$ radiation provided with a proportional counter. A scanning speed of $2 \theta / \mathrm{min}$. and a chart speed of $10 \mathrm{~mm} / \mathrm{min}$. was used in all cases. Transmission electron microscopy (TEM) images with selected area electron diffraction (SAED) 
pattern were recorded using (JEOL- JEM-1230) and accelerating voltage of 100 $\mathrm{kV}$ to get excellent imaging capabilities suitable for materials science applications. The prepared samples for TEM observation were dispersed in ethanol followed by ultrasonic vibration for $20 \mathrm{~min}$, then one drop is placed onto a copper grid coated with a layer of amorphous carbon. For UV/Vis measurement, PG Instrument CO., Ldt UV/Vis double beam spectrophotometer (T80+) was used to record the UV-Visible absorption spectra of the as-prepared particles.

\section{Results and Discussion.}

\subsection{X-ray Powder Diffraction}

The obtained XRD spectra were given in (Figs. $1 \mathrm{a}, \mathrm{b}$ and c) for the prepared samples using aqueous solutions of lithium, sodium and potassium hydroxides respectively. The X-ray diffraction patterns show that all $\mathrm{CuO}$ samples are of monoclinic tenorite structure. The XRD peaks of the copper oxide are in consistent with the data of the ICSD (Inorganic Crystal Structure Database) file, reference code (01-080-0076 [7], 00-048-1548 [8]. The peaks at $2 \theta$ values of $32.459,35.52,38.673,48.816,53.522,58.136,61.517,66.178$, $68.097,72.300,75.179$ correspond to the crystal planes of $110,11 \overline{1}, 111,20 \overline{2}$, $020,202,11 \overline{3}, 310,220,311,22 \overline{2}$ of crystalline copper oxide, respectively. Which are in good agreement with recent observations $[9,10,11]$.

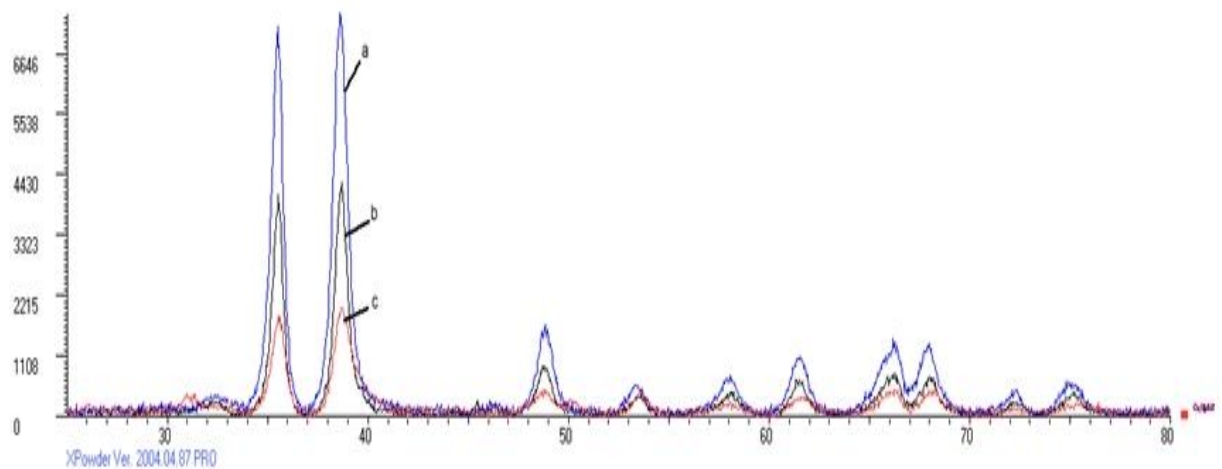

Fig. (1): $\mathrm{XRD}$ spectra of prepared $\mathrm{CuO}$ nanostructures by using $\mathrm{LiOH}, \mathrm{NaOH}$ and $\mathrm{KOH}$ ( $\mathrm{a}, \mathrm{b}$ and $\mathrm{c}$ respectively)

In the absence of microstrains, the grain size of powder can be determined from the Scherrer equation [6] $d=\frac{0.941 \lambda}{\beta \cos \theta}$ where $\lambda$ is the X-ray wavelength, $\beta$ is the full width at half maximum (FWHM) of the peak and $\theta$ is the Bragg angle, In order to estimate the microstrains, one should apply the well known WilliamsonHall equation [12]. 


$$
\beta \cos \theta=4 \varepsilon \sin \theta+\frac{k \lambda}{D}
$$

where $\theta$ is the diffraction angle, $\mathrm{k}$ the shape factor, $\lambda$ the wavelength of $\mathrm{X}$-ray, $\varepsilon$ is the microstrains of the crystal lattice and D is the particle size. Table (1) shows the grain size and microstrains of the prepared samples estimated from Scherrer equation and Williamson-Hall plot together with calculated lattice constants in nanometer and Degree of crystallinity.

Figure 2 shows the Williamson-Hall plot of the prepared $\mathrm{CuO}$ samples. The estimated values of both $\varepsilon$ and $\mathrm{D}$, calculated from the above equation, are listed in Table 1. The obtained data reveal that both particle size and micro-strain increases by increasing the alkalinity of the alkali metals ( $\mathrm{Li}, \mathrm{Na}$ and $\mathrm{k}$ ). TEM images of the prepared samples show in Fig.(3, 4, and 5), where rod like structure is observed. In addition the TEM images shows collates of the formed rods.

Table (1): Grain size and microstrains of the prepared samples estimated from Scherrer equation and Williamson-Hall plot together with calculated lattice constants in nanometer and Degree of crystallinity.

\begin{tabular}{|c|c|c|c|c|c|c|c|c|c|c|}
\hline \multirow{3}{*}{$\begin{array}{c}\text { Stabilizing } \\
\text { agent }\end{array}$} & \multirow{2}{*}{\multicolumn{2}{|c|}{$\begin{array}{l}\text { TEM Particle } \\
\text { size }(\mathrm{nm})\end{array}$}} & \multirow{3}{*}{$\begin{array}{c}\text { Band } \\
\text { gap }(e V)\end{array}$} & \multicolumn{3}{|c|}{ x-ray Particle size } & \multicolumn{3}{|c|}{$\begin{array}{l}\text { Lattice Constants } \\
(\mathrm{nm})\end{array}$} & \multirow{3}{*}{$\begin{array}{c}\text { D.O.C } \\
\%\end{array}$} \\
\hline & & & & \multirow{2}{*}{$\begin{array}{c}\text { Scherrer's } \\
(\mathrm{nm})\end{array}$} & \multirow{2}{*}{$\begin{array}{c}\text { Williams } \\
\text { on-Hall }\end{array}$} & \multirow{2}{*}{ Strain } & \multirow{2}{*}{$\mathrm{a}$} & \multirow{2}{*}{ b } & \multirow{2}{*}{$\mathrm{C}$} & \\
\hline & Breadth & Length & & & & & & & & \\
\hline $\mathrm{LiOH}$ & $4-11$ & $72-76$ & 2.09 & $16.7 \mathrm{~nm}$ & 20.2041 & 0.00234 & 0.469 & 0.344 & 0.512 & 59 \\
\hline $\mathrm{NaOH}$ & $6-15$ & $50-64$ & 2.28 & $18.02 \mathrm{~nm}$ & 31.0067 & 0.00265 & 0.469 & 0.343 & 0.512 & 78 \\
\hline $\mathrm{KOH}$ & $3-7$ & $11-76$ & 2.64 & $37.3 \mathrm{~nm}$ & 41.6216 & 0.00424 & 0.467 & 0.343 & 0.512 & 69 \\
\hline
\end{tabular}
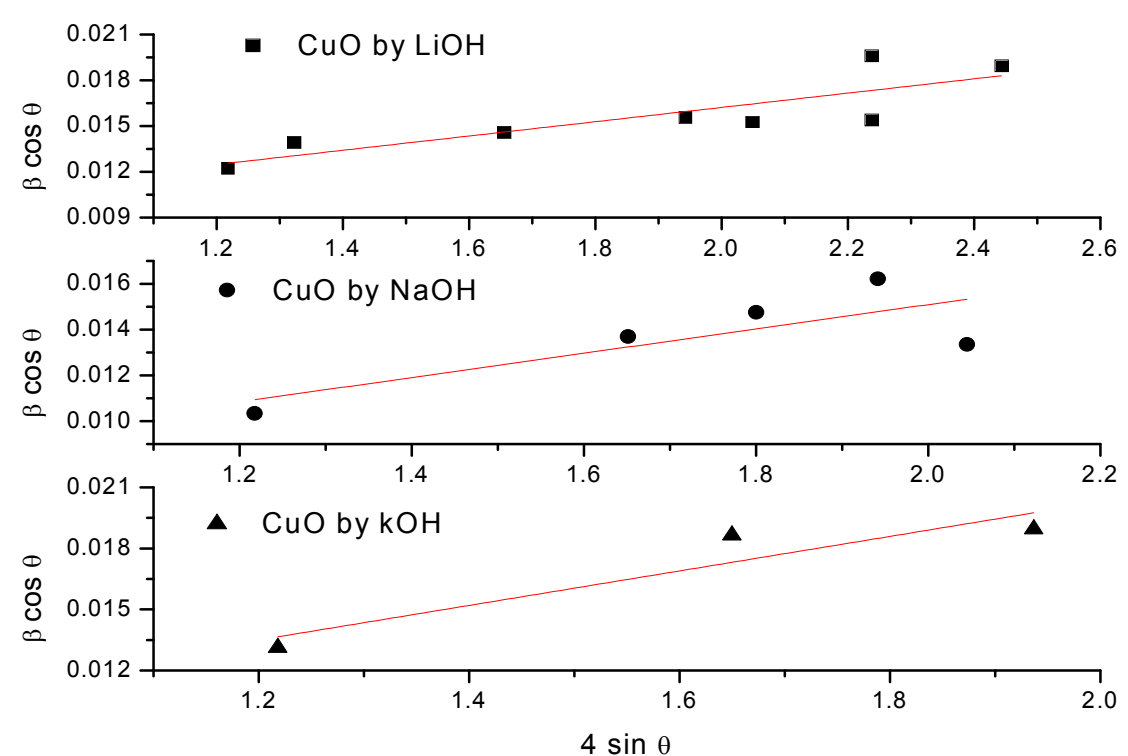

Fig. (2): Plot of $\beta_{h k l} \cos \theta$ vs. $4 \sin \theta$ of the prepared $\mathrm{CuO}$ samples. 


\subsection{Transmission electron microscopy}

The morphological surface characterization of the prepared samples were carried out by the transmission electron microscopy (TEM) equipped with the selected area electron diffraction (SAED) pattern. TEM images (Figs. 3 - 5) indicate the nanostructure of $\mathrm{CuO}$ prepared using lithium and sodium hydroxides grown as nanorods morphology. It is clear from (fig. 3) that $\mathrm{CuO}$ nanorods prepared by using lithium hydroxide solution were clustered to form collate nanorods with breadth ranging from $4 \mathrm{~nm}$ to $11 \mathrm{~nm}$ with length ranging from 72 to $76 \mathrm{~nm}$. However, the prepared sample by using $\mathrm{NaOH}$ solution (Fig. 4) shows $6-15 \mathrm{~nm}$ breadth and $50-64 \mathrm{~nm}$ length. Moreover, in the case of potassium hydroxide the breadth ranging from 3 to $7 \mathrm{~nm}$ length of $11-76 \mathrm{~nm}$ as shown in Fig. (5). Diffraction rings based on SAED images matches with the peaks in XRD patterns which proves the monoclinic structure of the prepared $\mathrm{CuO}$ particles.
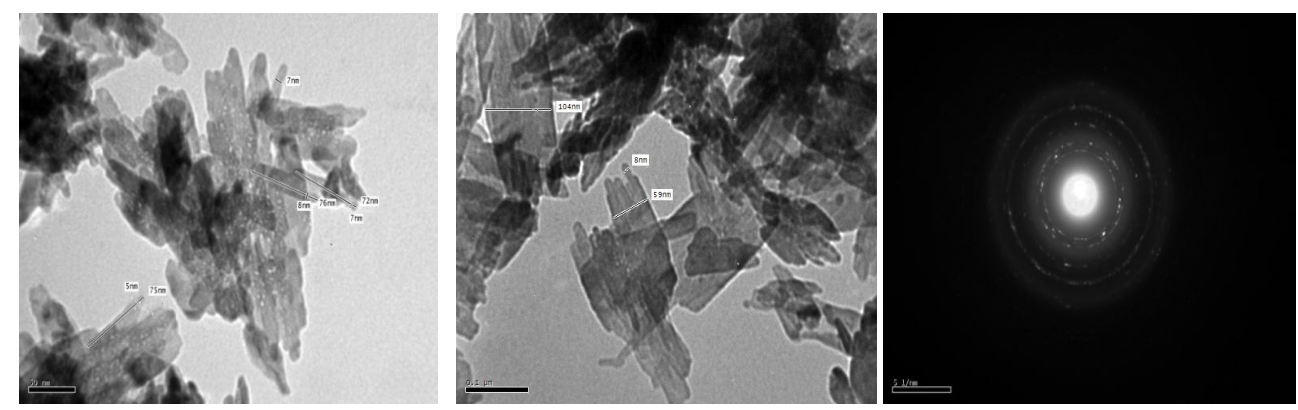

Fig. (3): Transmission electron microscopy (TEM) images of $\mathrm{CuO}$ prepared with the use of lithium hydroxide stabilizing agent together with selected area electron diffraction pattern.
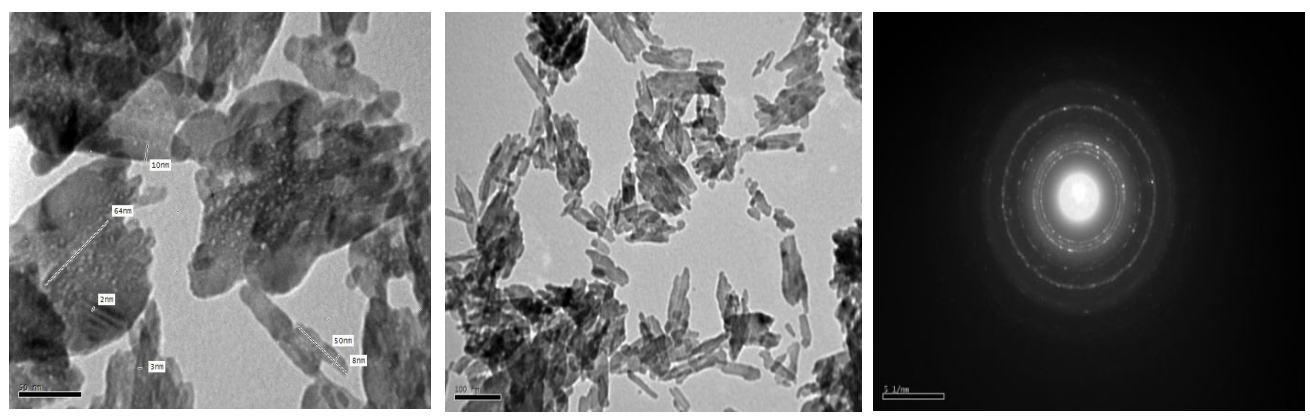

Fig. (4): Transmission electron microscopy (TEM) images of $\mathrm{CuO}$ prepared with the use of sodium hydroxide stabilizing agent together with selected area electron diffraction pattern. 


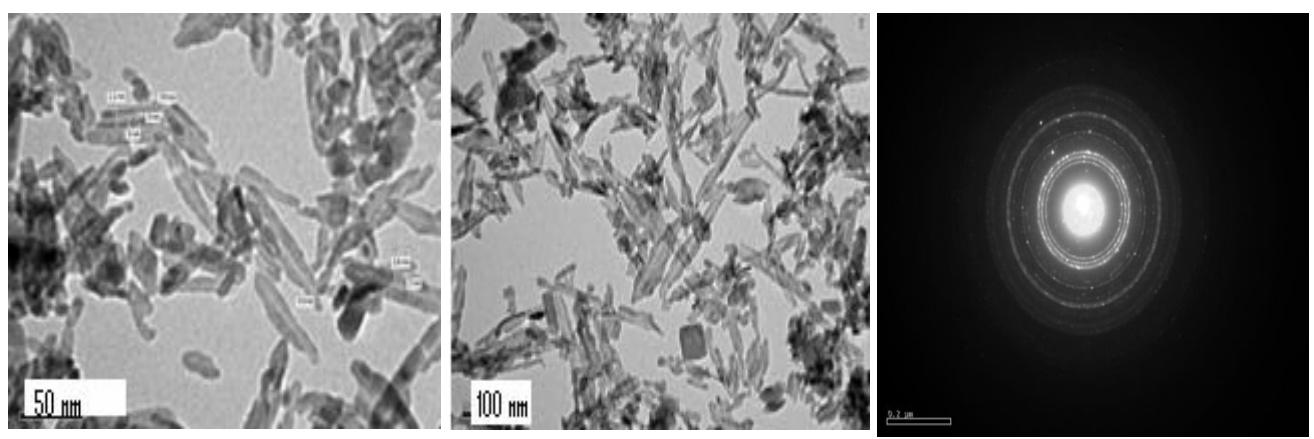

Fig. (5): Transmission electron microscopy (TEM) images of $\mathrm{CuO}$ prepared with the use of potassium hydroxide stabilizing agent together with selected area electron diffraction pattern.

\subsection{Optical absorption spectra}

UV-Vis absorption spectra of the prepared $\mathrm{CuO}$ samples were analyzed and presented in (Fig. 6) where the absorption peaks clearly observed at $218 \mathrm{~nm}$, $220 \mathrm{~nm}$ and $222 \mathrm{~nm}$ for $\mathrm{CuO}$ nanostructures prepared by $\mathrm{LiOH}, \mathrm{NaOH}, \mathrm{KOH}$ respectively which attributes to direct transitions according to M. L. Cohen, et.al[13]. A straight line is obtained for all prepared samples (Fig. 7) by plotting $(\alpha h v)^{2}$ versus $h v$ based on the relation $\alpha \mathrm{h} v=\mathrm{A}\left(\mathrm{h} v-\mathrm{E}_{\mathrm{g}}\right)^{\mathrm{n} / 2}$, where $\alpha$ is the absorption coefficient, $\mathrm{A}$ is constant (independent on $v$ ) and $\mathrm{n}$ is the exponent depending on quantum selection rules for the particular material. This indicates that, the absorption edge is due to a direct allowed transition, such transitions are most probable in crystalline materials due to translation symmetry. Table (1) shows that the obtained band gaps of the prepared samples together with the particles size and shapes. It is clear also that the obtained band gaps are larger than the recorded value for the bulk $\mathrm{CuO}(\mathrm{Eg}=1.2 \mathrm{eV})[14]$ which is related to the decrease of particle size and hence the quantum confinement. The obtained Eg values are in fair agreement with the data obtained by Rakhshani [13] and Wang [15]. 


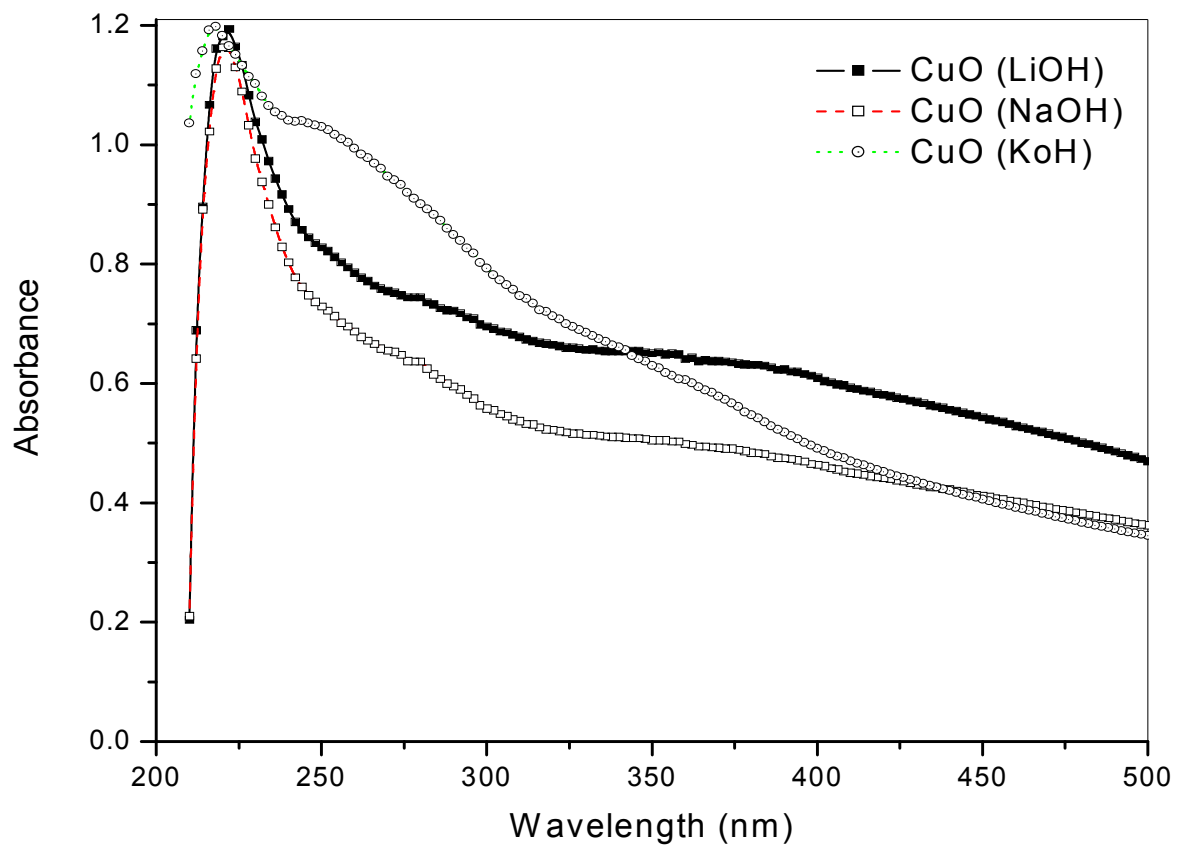

Fig. (6): UV-vis absorption spectra of nanostructure $\mathrm{CuO}$ prepared with different stabilizing agents.

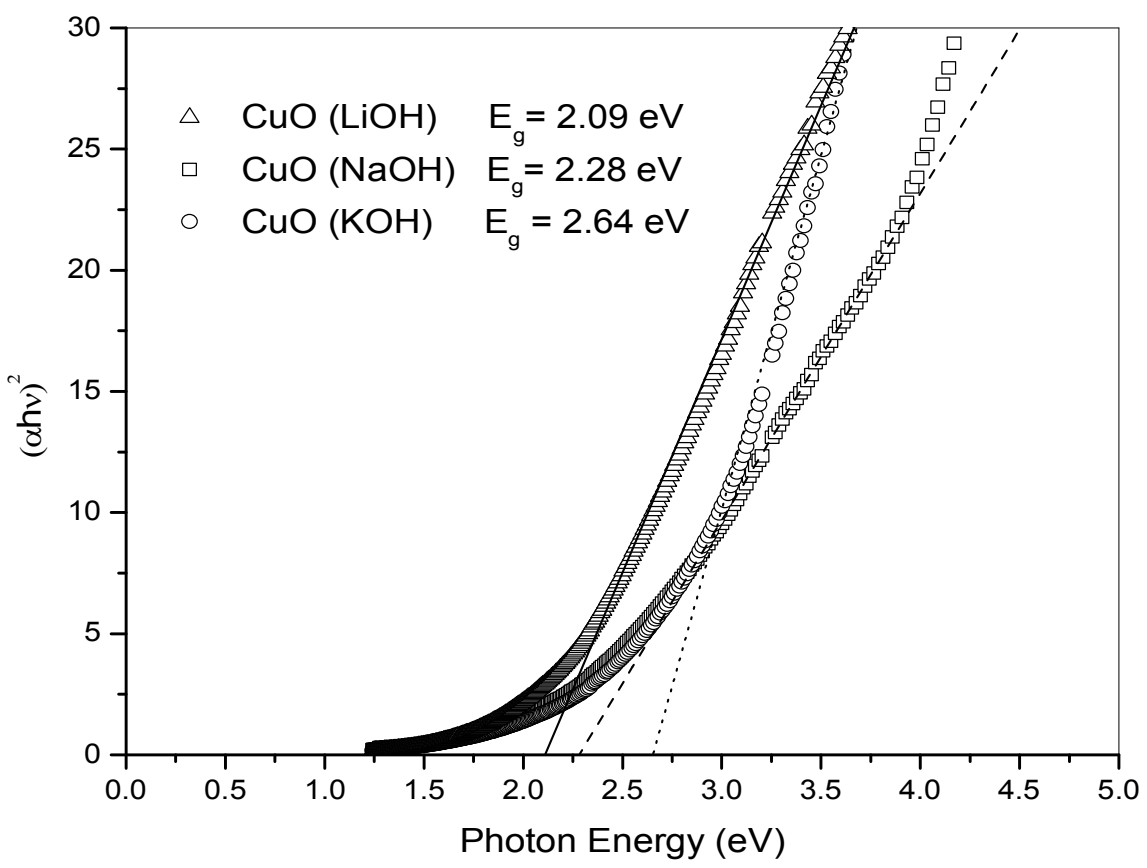

Fig. (7): Tauc plot for $\mathrm{CuO}$ nanostructures prepared from different solutions 


\section{References}

1. Hannink R. H. J. and Hill A. J. "Nanostructure control of materials" Woodhead Publishing Limited, Cambridge, (2006).

2. R. Nagarajan, T. Alan Hatton, "Nanoparticles: Synthesis, Stabilization, Passivation, and Functionalization, ACS Symposium Series", American Chemical Society, Oxford University Press. 996, (2008).

3. Wang Hui, Xu Jin-Zhong, Zhu Jun-Jie, Chen Hong-Yuan, Journal of Crystal Growth, V 244, P 88 (2002).

4. Lee Jusang and Gouma Pelagia I., (2010), Journal of Nanomaterials, V., P 1 (2011).

5. S.M. Lee, S.N. Cho, J.W. Cheon, Adv. Mater., V. 15 (5), P 441 (2003).

6. Yadav Raghvendra S., Pandey Avinash C., (2008), Physica E, V 40, P 660.

7. O. Garcia-Martinez, R.M. Rojas, E. Vila, Martin de, J.L.Vidales, Solid State Ionics, V 63, P 442 (1993).

8. J. Langford, D. Louer, J. Appl. Crystallogr., V 24, P 149 (1991).

9. Jenna Pike, Chan Siu-Wai, Zhang Feng, Wang Xianqin, Hanson Jonathan, Applied Catalysis A: General V 303, P 273 (2006).

10. Zhou Kebin, Wang Ruipu, Xu Boqing, and Yadong Li, Nanotechnology, V 17, P 3939 (2006).

11. Yang Zeheng, $\mathrm{Xu}$ Jun, Zhang Weixin, Liu Anping, and Tang Shupei, Journal of Solid State Chemistry, V 180, P 1390 (2007).

12. Mote VD, Purushotham Y. and Dole BN, Journal of Theoretical and Applied Physics, 6:6, p 1 (2012).

13. Cohen M.L. and Chelikowsky J.R., Electronic Structure and Optical Properties of Semiconductors, Springer -Verlag (1989).

14. A.E. Rakhshani, Solid-State Electron, V 29, P 7 (1986).

15. Wang Hui., Journal of Crystal Growth, V 244, P 88 (2002). 